\title{
Anomalous Viscosity Reduction and Hydrodynamic Interactions of Polymeric Nanocolloids in Polymers
}

\author{
Nafisa Begam, ${ }^{\dagger}$ Sivasurender Chandran ${ }^{\ddagger}{ }^{\ddagger}$ M Sprung, ${ }^{\complement}$ and J. K. Basu ${ }^{* \dagger}$ \\ Department of Physics, Indian Institute of Science, Bangalore, 560 012, India, Institute of \\ Physics, Albert-Ludwig-University of Freiburg, 79104 Freiburg, Germany, and Deutsches \\ Elektronen Synchrotron DESY, Notkestresse 85, 22607 Hamburg, Germany \\ E-mail: basu@physics.iisc.ernet.in
}

\begin{abstract}
One of the central dogma of fluid physics is the no-slip boundary condition whose validity has come under scrutiny, especially in the fields of micro and nanofluidics. Although various studies show the violation of the no-slip condition its effect on flow of colloidal particles in viscous media has been rarely explored. Here we report unusually large reduction of effective viscosity experienced by polymeric nano colloids moving through a highly viscous and confined polymer, well above its glass transition temperature. The extent of reduction in effective interface viscosity increases with decreasing temperature and polymer film thickness. Concomitant with the reduction in effective viscosity we also observe apparent divergence of the wave vector dependent hydrodynamic interaction function of these colloids with an anomalous power law

\footnotetext{
*To whom correspondence should be addressed

${ }^{\dagger}$ Indian Institute of Science

¥Albert-Ludwig-University of Freiburg

`Deutsches Elektronen Synchrotron DESY
} 
exponent of $\sim 2$ at the lowest temperatures and film thickness studied. Such strong hydrodynamic interactions are not expected for polymeric colloidal motion in polymer melts. We suggest hydrodynamics, especially slip present at the colloid-polymer interface which determines the observed reduction in interface viscosity and presence of strong hydrodynamic interactions.

\section{Introduction}

It is widely accepted that hydrodynamic interactions (HI) are strongly screened to within a segmental length in polymer melts. ${ }^{1-3}$ The dynamics of unentangled polymer melts and short time dynamics of entangled melts, to a large extent, depend on this screening. ${ }^{4}$ However, recent reports ${ }^{5-12}$ have shown that the hydrodynamic interactions can be enhanced in reduced dimension of polymers and colloidal systems with long range interactions. One of the primary aim of such studies is to help improve our understanding of the Physics of fluids at the micro and nanoscales. ${ }^{13,14}$ Especially studies related to interfacial hydrodynamics and the validation of the well known mostly boundary conditions at the nanoscale have began to emerge. ${ }^{14-17}$ While hydrodynamic slip has been observed in artificially engineered superhydrophobic surfaces ${ }^{14}$ or for polymer thin films on polymer brush surfaces ${ }^{15-17}$ a clear microscopic understanding of such observations is still elusive. Although some studies have emerged, recently, on colloidal and nanoparticle diffusion in polymer solutions and melts, considering the diverse applications of such systems, ${ }^{18-21}$ there has been surprisingly few ${ }^{19}$ efforts to study hydrodynamic interactions between colloidal particles in polymer solutions and melts. In particular, thin films of polymer nanocomposites (PNCs) having polymer grafted nanoparticles (PGNPs) dispersed in polymer matrix provide an effective system to study dynamics of such nano colloids in visco-elastic media under controlled confinement. ${ }^{20}$ Various aspects like dispersion and thermo-mechanical properties have been widely studied in such thin PNC films. ${ }^{22-27}$ However, dynamics of the embedded nanoparticles, which could determine the state of dispersion and related physical properties of the blend has not 
been widely studied. We have shown recently, ${ }^{28}$ that confinement enhances dispersion of PGNPs in thin polymer films, with the extent of enhancement depending on the nature of the PGNP-matrix polymer interface, which eventually also determines the glass transition temperature, $T_{g}$, of the blend. More specifically, it is widely believed that a dewetting PGNP-matrix polymer interface leads to reduction of $T_{g}$ of the PNC while a wetting interface leads to an enhancement of the same with respect to the neat blend. ${ }^{22,25}$ Here, we report measurements of microscopic dynamics of PGNPs in thin polymer films of various thickness as a function of temperature and scattering wave vector, $q_{x}$. We observe evidence of large reduction in the effective viscosity $\left(\eta_{e f f}\right)$ experienced by these PGNPs in the thin films. For films with smaller thickness, $h$, we observe larger reduction in effective viscosity with decreasing temperature, approaching the matrix polymer $T_{g}$. This suggests the presence of an interface viscosity, $\eta_{s}$, dominated, possibly, by slip at the PGNP-matrix polymer interface. We also find evidence for the presence of strong HI on dynamics of nanocolloids as quantified with an anomalous $q_{x}$ dependent hydrodynamic interaction function, $H\left(q_{x}\right) \sim q_{x}^{-\alpha}$, where 1 $\leq \alpha \leq 2$ depending on temperature and film thickness. Hydrodynamic slip at the dewetting PGNP-polymer interface, relevant to our chosen systems, is indicated as the likely cause for both the reduction in effective interface viscosity and the unique power law scaling of $H\left(q_{x}\right)$, observed here. Our results throw light, not only on the rich Physics at play in dynamics of these polymeric nano colloids in confined polymer melts but also highlights how this could be crucial in determining their dispersion and hence all relevant physical properties in such PNC films.

\section{Experimental details}

The experimental results presented here are based on thiol terminated polystyrene(PST, molecular weight, $M_{w}=3.2 \mathrm{kDa}$ ) grafted gold (Au) nanoparticles (PGNPs) of core diameter, $\sim 3.9 \mathrm{~nm}$ and a grafted shell thickness of $1.1 \mathrm{~nm}$, synthesized following methods used 
earlier. ${ }^{28}$ PGNPs and matrix polystyrene (PS) $\left(M_{w}=90 \mathrm{kDa}\right.$ and Radius of gyration $R_{g}=$ $8 \mathrm{~nm}$ ) were dissolved in toluene separately and stirred well for a long time (24 hours) to get homogeneous solutions. These two solutions were then mixed in a certain ratio to obtain $0.3 \%$ volume fraction of gold particle in polymer. This final polymer nanocomposite (PNC) solution was stirred for long time (24 hrs) to ensure homogeneous dispersion of particles. Films of this PNC of thicknesses 65 and $39 \mathrm{~nm}$ were spin coated (Apex Instrument, India) on silicon substrates. The films were annealed at $150^{\circ} \mathrm{C}$ (well above $T_{g}$ of PS, which is around $100^{\circ} \mathrm{C}$, measured from differential scanning calorimetry) in vacuum of $5 \times 10^{-3}$ mbar for 12 hrs to remove the trapped solvent.

X-ray photon correlation spectroscopy (XPCS) measurements in grazing incidence x-ray scattering (GIXS) geometry were performed at the beamline P10 of PETRA III, DESY, Hamburg, Germany. The beam size was $25 \times 25 \mu m^{2}$ with an incident energy of $8 \mathrm{KeV}$ in vacuum of $\sim 10^{-4}$ mbar. The scattered intensity was recorded by Maxipix detector in $2 \mathrm{X} 2$ configuration (pixel size of $55 \times 55 \mu \mathrm{m}^{2}$ ). The exposure time for each frame was 0.5 second and typically around 1000 frames were collected in each measurements. After each measurement the sample was shifted laterally across the beam by $\sim 50 \mu \mathrm{m}$ to avoid radiation damage. Stability of intensity over time is checked for any systematic increase or decrease, which is indicative of radiation damage or any other issues related to thermal drift. The measurements were done at two different incident angles $(\theta)$, slightly below $\left(0.15^{\circ}\right)$ and above $\left(0.168^{\circ}\right)$ the critical angle $\left(\sim 0.16^{\circ}\right.$ at the energy of the incident X-rays) of the PNC film. Penetration depth of X-ray into the film at this energy is $\sim 13 \mathrm{~nm}$ for an incident angle of $0.15^{\circ}$ and hence measurements at this incident angle is sensitive to the surface part of the film. On the other hand for incident angle $0.168^{\circ}$, X-ray penetrates the entire film and probes the entire dynamics of the film. Hence we denote the former configuration as surface $\left(\theta=0.15^{\circ}\right)$ and latter as bulk $\left(\theta=0.168^{\circ}\right)$ configuration..$^{29,30}$ Measurements were performed for a range of temperatures above the $T_{g}$ of the samples.

XPCS measurements provides the intensity autocorrelation function, $g_{2}\left(q_{x}, t\right)$ which is given 
by $^{31-33}$

$$
g_{2}\left(q_{x}, t\right)=1+b\left|f\left(q_{x}, t\right)^{2}\right|
$$

where $f\left(q_{x}, t\right)$ is intermediate scattering function (ISF), $b$ is an instrumental factor called the speckle contrast, $t$ is delay time. $f\left(q_{x}, t\right)$ has the general time dependent functional form

$$
f\left(q_{x}, t\right)=\exp \left[-(t / \tau)^{\beta}\right]
$$

where $\tau$ and $\beta$ are the relaxation time and Kohlrausch exponent, respectively. In the limit of short time relaxation this function can be approximated as

$$
f\left(q_{x}, t\right)=\exp \left(-q_{x}^{2} D_{s} t\right)
$$

where $D_{s}$ is the short time diffusion coefficient which can be extracted from the slope of $\ln f\left(q_{x}, t\right)$ vs $t$ plot.

In the presence of $\mathrm{HI}$, the short time $q_{x}$ dependent diffusion coefficient, $D_{s}\left(q_{x}\right)$ can be written as. ${ }^{34-36}$

$$
D_{s}\left(q_{x}\right)=\frac{D_{0} H\left(q_{x}\right)}{S\left(q_{x}\right)}
$$

where $D_{0}$ is the diffusion coefficient given by the Stokes-Einstein relation for a particle of radius, $R$, moving in a medium of viscosity, $\eta, H\left(q_{x}\right)$ is the $q_{x}$ dependent hydrodynamic interaction function and $S\left(q_{x}\right)$ is the static structure factor of PGNPs dispersed in the polymer matrix. If the static structure factor is known the form of HI can be easily determined.

\section{Results and Discussions}

Figure 1 shows the variation of ISF for measurements in the bulk configuration for two representative temperatures and wave vectors for both the $65 \mathrm{~nm}$ and $39 \mathrm{~nm}$ films. Similar 
data for the surface configuration measurements have also been presented in SM Fig. $4 .{ }^{37}$ What is striking is the obvious difference in both temperature and wave vector dependence of the $39 \mathrm{~nm}$ data from the $65 \mathrm{~nm}$ data. The $65 \mathrm{~nm}$ data clearly shows decrease in $\tau$ with increasing temperature and $q_{x}$ whereas the $39 \mathrm{~nm}$ data seems to suggest an independence with both temperature and $q_{x}$. This clearly indicates existence of intriguing dynamics of the PGNPs in the PS films, especially with increasing confinement. Similar $q_{x}$ dependence for $\tau$ is also observed for the thinnest $18 \mathrm{~nm}$ film which is shown in SM Fig. $5 .{ }^{37}$ Figure 2 shows

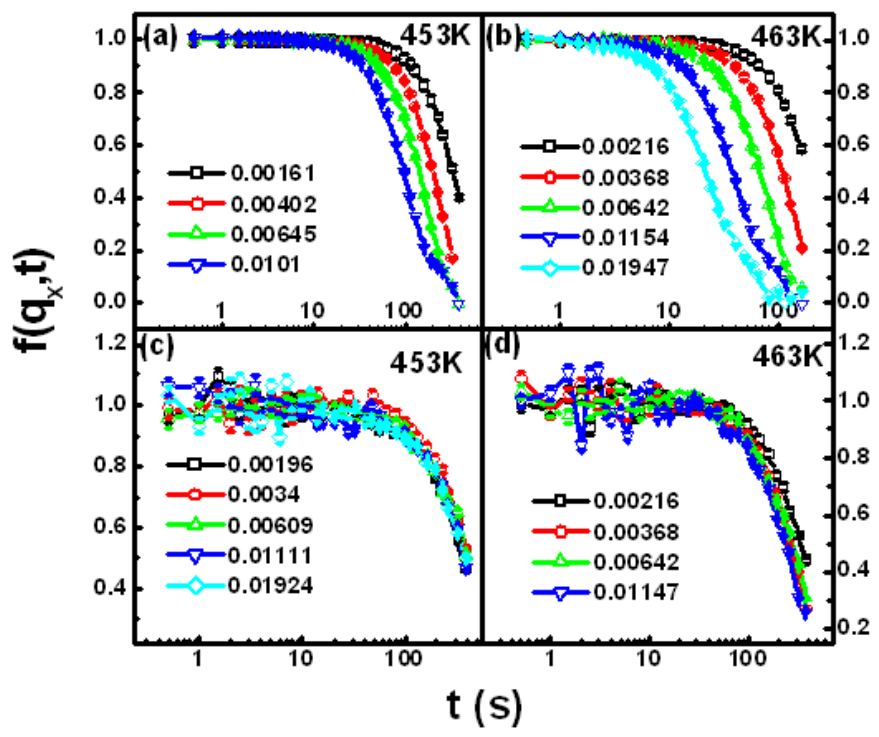

Figure 1: Typical ISF, $f\left(q_{x}, t\right)$ for bulk and surface configurations of 65 (a,b), $39 \mathrm{~nm}(\mathrm{c}, \mathrm{d})$ films is shown for two representative temperatures (as indicated in the figure). The unusual $q_{x}$ dependence of ISF for $65 \mathrm{~nm}$ film and $q_{x}$ independence of ISF for $39 \mathrm{~nm}$ film can be noticed.

the $q_{x}$ dependent relaxation times, $\tau$, extracted from the $f\left(q_{x}, t\right)$ data using Eqn. (2), for all the films at various temperatures as indicated in the panels. The relaxation time, expectedly, decreases with temperature for both bulk and surface configurations for the $65 \mathrm{~nm}$ film (Fig. 2(a)-2(b)). The $q_{x}$ dependence of $65 \mathrm{~nm}$ films evolves with temperature and becomes almost $q_{x}$ independent at the lowest measured temperature. There is not much difference between surface and bulk relaxation times which is along the expected lines unlike some earlier 


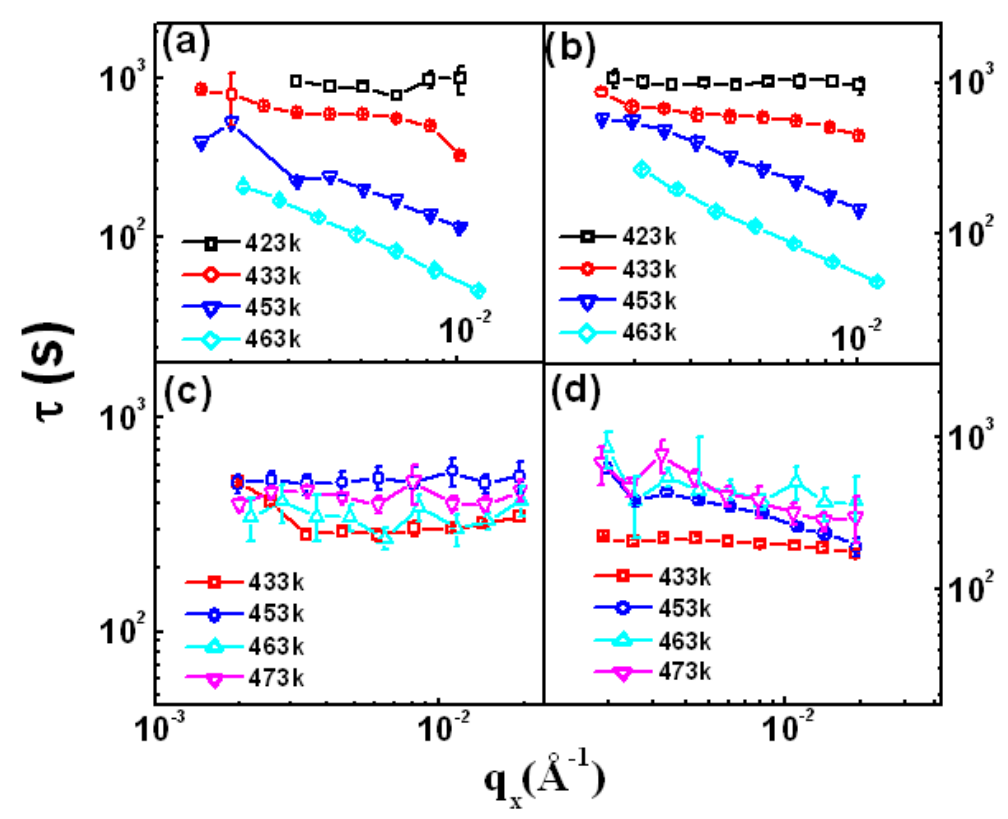

Figure 2: Wave vector $q_{x}$ dependent relaxation time $\tau$ is shown for films of thickness, 65 $(\mathrm{a}, \mathrm{b})$ and $39 \mathrm{~nm}(\mathrm{c}, \mathrm{d})$ in both bulk (left) and surface (right) configurations and at various temperatures as indicated in the figure. Anomalous evolution of $\tau$ with both $q_{x}$ and temperature could be noticed.

reports. ${ }^{30}$ For the $39 \mathrm{~nm}$ films (Fig. 2(c)-(d)), $\tau$ indicates both anomalous temperature and $q_{x}$ dependence at all measured temperature. Similar behavior with the variation of $q_{x}$ is observed for the $18 \mathrm{~nm}$ films (SM Fig. 6). In the following we first try to address the observed anomalous temperature dependence of $\tau$. The observed temperature independence of $\tau$ is contrary to the expected variation of relaxation time due to the change in viscosity of polymers with temperature. To proceed, we make the reasonable assumption that $\tau \sim \frac{1}{D}$, where $D$ is the diffusion coefficient of PGNPs. Further, assuming validity of Stokes-Einstein (SE) relation, which is expected to hold at measurement temperatures which are $\sim 50-60$ $\mathrm{K}$ above $T_{g}$, as has been shown earlier for thin polymer films, ${ }^{33}$ we can write $\tau \sim \frac{6 \pi \eta R}{K_{B} T}$, where $K_{B}$ is the Boltzmann constant and $T$ is the temperature. We now use the well known temperature dependence of bulk viscosity of $\mathrm{PS}^{38}$ to obtain the expected viscosity of the PNC films at each temperature for all the films. Although, existence of confinement effects on viscosity of thin polymer films has been widely discussed with a great deal of controversy ${ }^{30,33}$ we have avoided usage of any such data or models. In any case we assume that any possible 
viscosity anomalies which exist in our films occur mostly at lower temperatures (near their $T_{g}$ ) and at the highest measured temperature at which our dynamics data exists, these anomalies would be minimal and it is, therefore, safe to assume bulk-like viscosity at the highest measured temperature. To quantify the anomaly in the temperature dependence of $\tau$, we plot the ratio $\frac{\tau_{i}}{\tau_{473}}$ as a function of temperature for both 65 and $39 \mathrm{~nm}$ films. This ratio is expected to have the same scaling as the corresponding ratio of bulk viscosities, $\frac{\eta_{i}}{\eta_{473}}$. As shown in Fig. 3(a), the normalized relaxation time shows apparently different scaling
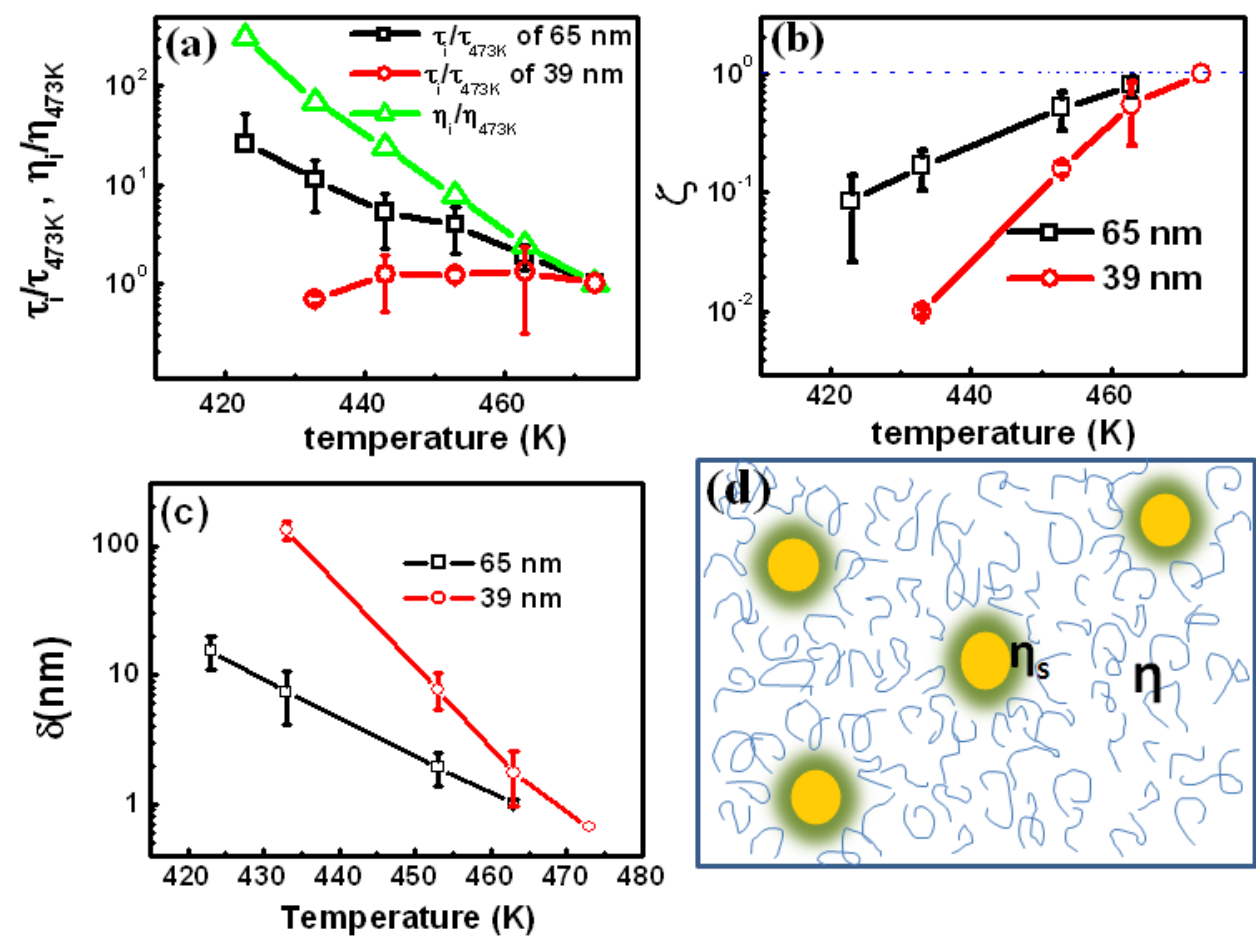

Figure 3: (a) Comparison of the renormalized relaxation times for both 65 and $39 \mathrm{~nm}$ film are shown in comparison with the expected scaling of renormalized bulk viscosity, (b) Evolution of $\varsigma$ as defined in eqn. 5 with temperature is shown for both 65 (open squares) and $39 \mathrm{~nm}$ (open circles) films. Horizontal line (dashed-dot) indicates the expected behavior, in the absence of interfacial effects on the dynamics of PGNPs.(c) Approximate slip length calculated showing the temperature and thickness dependence. (d) Schematic describing the presence of different viscosities viz., at the interface $\left(\eta_{s}\right.$, driven by the dewetting PGNPpolymer interface) and for the matrix $(\eta)$ is shown.

from normalized bulk viscosity exemplifying the temperature dependent anomaly in PGNP dynamics.

The temperature dependence of $\tau$ and its deviation from the expected temperature de- 
pendence of bulk viscosity, $\eta$, of PS suggests the existence of an effective viscosity, $\eta_{e f f}$, experienced by the PGNPs in the PS thin films which is different from $\eta$. To quantify this viscosity anomaly and $\eta_{\text {eff }}$ we define a viscosity anomaly parameter, $\varsigma$, as

$$
\varsigma=\frac{\tau_{i}}{\tau_{473}} / \frac{\eta_{i}}{\eta_{473}} \sim \frac{\eta_{e f f}}{\eta} \sim \frac{\eta_{s}}{2 \eta_{s}-\eta}
$$

which characterizes the viscosity anomaly experienced by the PGNPs in their motion through the polymer melt. More generalized form of this equation is shown in the supporting information. ${ }^{37}$ Here, $\eta_{s}$, is the effective interface viscosity at the PGNP-PS interface (refer Fig 3(d)). Let us now discuss the implications of eqn. 5 and how it could help understand the microscopic model underlying the observed viscosity anomaly. From eqn. 5 above, it becomes clear that in the absence of any viscosity anomalies the two ratios (viscosity and $\tau$ ) should have similar temperature dependence and hence $\varsigma$ should be 1 at all temperatures. However, as Fig 3(b) shows, this is clearly not the case. The viscosity anomaly parameter increases with decreasing temperature approaching the PS $T_{g}$. Further, the anomaly is considerably larger for the thinner films. The existence of such effective nanoscale viscosity could be postulated based on few models which we briefly discuss below. de Gennes and Wyart (DW $)^{39}$ proposed the existence of a length scale dependent viscosity in polymer melts which could be tested by exploring the diffusivity of colloids of various sizes, especially those comparable or smaller than the polymer entanglement length, in melts. Experimentally, the DW predictions were validated by Mackay et al $^{40}$ and few others ${ }^{36,41,42}$ in terms of anomalous increase in nanoparticle diffusivity, compared to predictions of SE relation. However, as Mackay et al have shown ${ }^{40}$ that, the large increase in diffusivity, and hence reduction in $\eta_{e f f}$, that was observed in their studies, were largely temperature independent. Clearly, therefore, the DW model and its recent, more detailed, numerical validations ${ }^{43,44}$ is not applicable in our case. We will elaborate other reasons as to why this class of model is not applicable for our data. The alternate class of models which has been applied to explain viscosity reduction or mobility enhancements in polymer melts (in certain cases in concen- 
trated solutions) postulates the existence of a layer of different viscosity (and in some cases density) at the polymer-substrate or polymer-colloid (or nanoparticle) interface, ${ }^{15,16,21,45-49}$ with or without slip. In this regard, we would also like to state that drag reduction of colloids and nanoparticles in Newtonian fluids, due to existence of hydrodynamic slip, at the fluid-solid or nanoparticle-fluid interface has also been reported, ${ }^{47,50}$ especially those dealing with Epstein drag. ${ }^{51,52}$ However, the magnitude of change in diffusivity or effective viscosity, in such cases is much smaller than what we observe here making the relevant expressions (for instance those involving various forms of the Knudsen parameter, $K_{n p}$, which has been discussed briefly in supporting information ${ }^{37}$ ) unusable in our case. More importantly, none of these models deal with, explicitly, the temperature dependence, which is so apparent in our case. The recent work of Wang and Hill $(\mathrm{WH}),{ }^{49}$ is of course, quite relevant to our case, especially since it deals with PGNPs in a polymer melt. The WH model discusses the origin of large negative intrinsic viscosity in PNCs ascribing it, largely, to the presence of an interface layer of polymer chains at the nanoparticle-polymer interface of viscosity and density different from the bulk. They claim to be able to explain the results of Mackay et $\mathrm{al}^{40}$ quite well using this interface viscosity based model and neglecting slip at this interface. However, the WH model, does not discuss any temperature dependence of the intrinsic viscosity and since it argues that it explains the result of Mackay et al, which as we have mentioned above, shows this intrinsic viscosity to be largely independent of temperature, we feel that this model would not quite capture the essence of our viscosity anomaly. However, we will discuss the aspect hydrodynamic slip in the WH model, which might be relevant to our system, without of course, explaining the temperarure dependent viscosity anomaly of our samples. We now discuss the model of Servantie and Muller (SMu) which discusses the presence of not only a layer with surface viscosity, $\eta_{s}$, at the polymer-solid interface but also discusses the presence of hydrodynamic slip at this interface which relates to this surface viscosity and, most importantly, predicts a temperature dependence as well. The SMu model predicts the slip length $\delta$ to increase with decreasing temperature, approaching, $T_{g}$, leading 
to enhancement in segmental mobility with decreasing temperature, contrary to what would be expected from the increased bulk viscosity with reduction in temperature. The fact that, in our case, $\eta_{e f f}$, increases with reducing temperature towards $T_{g}$ is, therefore, strongly suggestive of slip at PS-PGNP interface as per the SMu model. ${ }^{48}$ Given the dewetting interface between the PGNP-PS interface, ${ }^{53}$ it is tempting to calculate the variation of corresponding slip length $\delta$ with temperature. Using the estimate of the effective interface viscosity, $\eta_{s}$ (SM Fig. 9), according to eqn. 5 , we have estimated $\delta$ for our system, using the approximate form $\delta \sim\left(\frac{1}{\eta_{s} / \eta}\right) \delta_{s}$ as suggested in ${ }^{48}$ where $\delta_{s}$ is microscopic slip length which we considered equal to the segmental length of PS. Figure 3 (c) shows the evolution of $\delta$ with temperature, which is similar to the variation predicted by Servantie et.al. ${ }^{48}$ Although there might be uncertainties in the actual magnitudes of extracted $\delta$ due to the approximations involved, including that in the microscopic slip length, $\delta_{s}$, the temperature and film thickness dependence should be reliable. Also, the values of $\delta$ are not too different from those extracted for polymers on a flat substrate. ${ }^{15,16}$ Although, the actual effective viscosity experienced by the PGNPs in the film could have various factors (including intrinsic viscosity gradients in PS films ${ }^{30}$ ), based on the above discussion, we postulate that it is largely due to the existence of an interface viscosity, $\eta_{s}$ (especially for $39 \mathrm{~nm}$ films), which is different from the bulk viscosity, $\eta$, as indicated in the schematic in Fig. 3(d). While the hydrodynamic slip at this interface enhances the mobility of the PGNPs with decreasing temperature, the increase in bulk PS viscosity with decreasing temperature tends to reduce the mobility and the observed $\tau$ is a resultant of these two competing effects which exists in our system. In, general, depending on the ratio of grafted to matrix polymer molecular weight, this interface viscosity and slip effect could be either absent or could act in a way to decrease the particle mobility further. It might be mentioned here that some initial temperature dependent viscosity estimates of films similar to that used here for XPCS studies as well as for a case where the grafted-to-matrix polymer molecular weight ratio is much higher, does seem to corroborate the trends suggested above (SM Fig 9). 
Hydrodynamic slip at a polymer melt-solid interface has been dealt with extensively ${ }^{15,16,46}$ over the last few decades, especially in thin polymer films. ${ }^{15,48}$ For the corresponding case of polymer near nanoparticle the role of the nanoparticle-polymer interface has been highlighted in the context of dispersion ${ }^{22,24,28,54}$ or thermo-mechanical properties of PNCs. ${ }^{23,25,54}$ However, elucidating its role in nanoparticle motion in PNCs and especially PNC thin films has not been addressed as effectively. ${ }^{39,43,49}$ As mentioned earlier, the WH model ${ }^{49}$ has delineated the role of morphology and hydrodynamic slip at the nanoparticle-polymer interface in determining the effective viscosity of PNC melts. We believe that the de-wetting nature of our PGNP-PS interface and the low penetrability ${ }^{53}$ by the matrix PS chains of the grafted chains makes the existence of large interface slip and an effective reduced viscosity at the nanoparticle-polymer interface highly probable. However, apart from the work of Wang et al explicit treatment of this aspect to nanoparticle-polymer melt interface has been rare. ${ }^{39,49}$ The extent of slip is dependent on the penetration length of the matrix chains into the flat brush region ${ }^{48,55}$ (in our case the PGNP particles). In fact the WH model ${ }^{49}$ suggests that slip becomes relevant in determining the intrinsic viscosity provided the width of the PGNPpolymer interface is smaller. This is actually quite relevant for our system of grafted PST and matrix PS chain molecular weight ratios for which this width is estimated ${ }^{53}$ to be quite small compared to the PGNP diameter. Interestingly though, Wang and Hill have compared their results to those of Mackay et al ${ }^{18}$ and have mostly de-emphasised the possibility of slip being an important parameter in determining the viscosity of PNCs, probably, because of the lack of experimental data presenting such a scenario. Since Wang and Hill do not treat the temperature dependence of viscosity in PNC, we use the analogy with SMu model. ${ }^{48}$ Our observation of larger temperature dependent viscosity anomaly with confinement, can, however, be understood, in terms of the polymer chain conformation under confinement as has been suggested earlier by us $^{28}$ and others. ${ }^{22}$ The suggested enhanced chain orientation at the interface with confinement, possibly, leads to the creation of a smoother interface between the matrix chains and the PGNPs. This results in even larger interface mobility between 
PGNP-matrix polymer interface and hence larger reduction in effective $\eta_{s}$ with confinement.

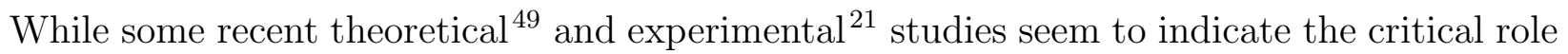
of the colloid-fluid interface, especially slip, in determining magnitude of effective interface viscosity or mobility, such large reduction in effective interface viscosity enhancements have neither been observed nor predicted.

We would like to state here that although, the dimensions of our system (PGNP diameter $\sim 6 \mathrm{~nm}$ and PS tube diameter $\sim 8-9 \mathrm{~nm})$ also satisfies the conditions for applicability of the DW model for non-Einstein like viscosity in our PNC films, the relatively weak temperature dependence of entanglement tube diameter suggests that the DW models or its analogues cannot explain the observed temperature dependent viscosity anomaly effects. Further reasons to believe that this model might not be applicable will become evident below.

Apart from the unusual temperature dependence, the $q_{x}$ dependence of $\tau$ is also anomalous, especially for the $39 \mathrm{~nm}$ films and also for $18 \mathrm{~nm}$ films (SM Fig. 6). In fact, careful inspection of Fig. 2(a), (b) for the $65 \mathrm{~nm}$ films, interestingly, shows a unique temperature dependent anomaly in the $q_{x}$ dependence of $\tau$ with the lowest temperature (423K) being almost independent of $q_{x}$. Here, again the DY model ${ }^{39}$ and the related detailed enumeration ${ }^{43,44}$ along the same lines, reveal a $\eta_{\text {eff }}$ scaling with $q_{x}$ as $\eta_{\text {eff }} \sim q_{x}^{2}$ which is clearly inconsistent with the $q_{x}$ dependence of our measured $\tau$ or $\eta_{e f f}$. To understand this behavior we have extracted $D_{s}\left(q_{x}\right)$ using Eqn. 3 (SM Fig. 9 and SM Fig. 10). ${ }^{37}$ The extracted $q_{x}$ dependent diffusion coefficient is indicative of presence of strong HI for PGNP motion with the PS films, which is quite unexpected due to the widely believed screening of $\mathrm{HI}$ in polymer melts. ${ }^{1-3}$ It is possible, though, that confinement could enhance HI in these films as seen in confined colloidal suspensions. ${ }^{6-10}$ We now turn our attention to the determination of the hydrodynamic interaction function, $H\left(q_{x}\right)$. To evaluate $H\left(q_{x}\right)$, we have calculated $S\left(q_{x}\right)$ (SM Fig. 12) from the static scattering profiles obtained for all the samples at all the temperatures at which the $g_{2}\left(q_{x}, t\right)$ profiles were also measured, as well as $D_{S}\left(q_{x}\right)$. The calculated $H\left(q_{x}\right)$ is shown 


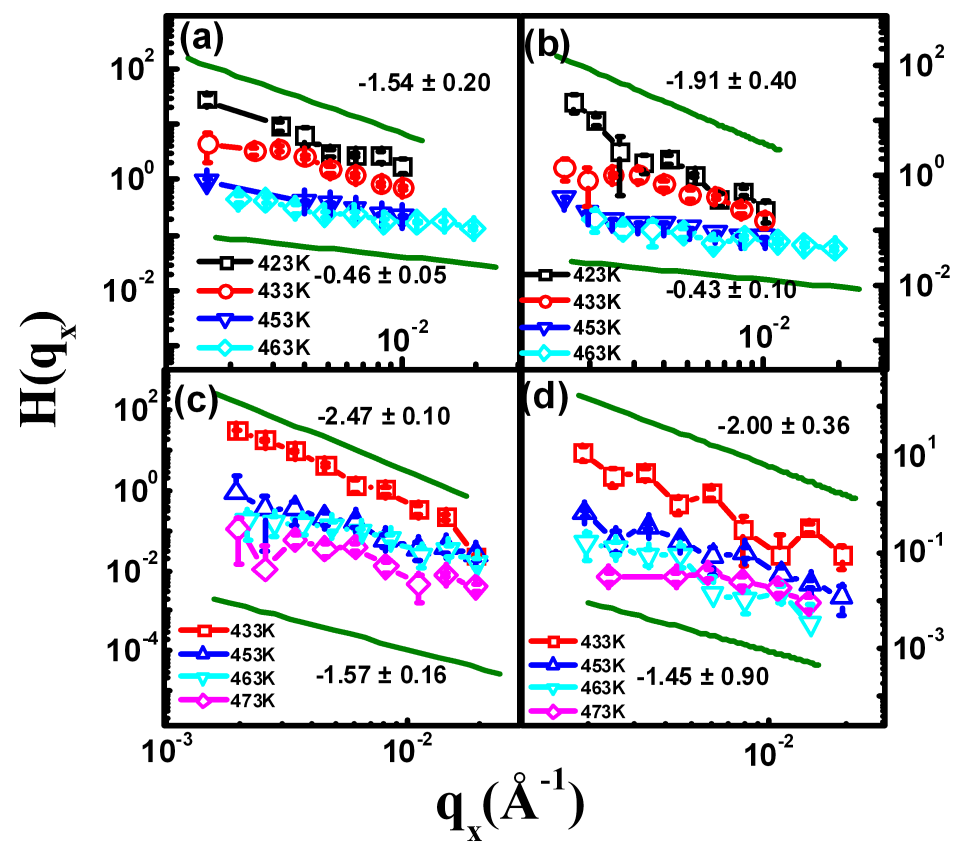

Figure 4: Wave vector $\left(q_{x}\right)$ dependent hydrodynamic interaction function of the polymeric colloids dispersed in polymer thin films of thicknesses 65 (a,b) and $39 \mathrm{~nm}$ (c,d) in both bulk (left) and surface (right) configurations. Solid lines in each panels show the exponent $\alpha$ for the lowest and highest temperatures studied, indicating the enhancement of hydrodynamic interactions with decreasing temperature and film thickness. 
in Fig. 4. Focusing first on the $65 \mathrm{~nm}$ data we find that $H\left(q_{x}\right)$ seems to show a power law decay similar to what has been reported recently for q2D colloidal monolayers. ${ }^{5,10}$ In the existing reports, what is interesting is that in one experimental configuration the exponent, $\alpha$, was reported to be $1^{6,10,11}$ and this is supported by some theoretical results ${ }^{5,11}$ while in another case it was measured to be $\sim 2$ from experiments. ${ }^{10}$ The exponents seem to be very sensitive to the nature of the hydrodynamic boundary conditions. Here we can observe an almost continuous increase of this exponent from $0.5-2$ with decreasing temperature. In fact this $q_{x}$ dependence of $H\left(q_{x}\right)$ or $D_{S}\left(q_{x}\right)$ (SM Fig. 10) explains the observed anomalous $q_{x}$ dependence of $\tau$ as shown in Fig. 2. The behavior is similar for the $39 \mathrm{~nm}$ data with $\alpha$ varying between $1.5-2.5$ in the measured temperature range indicating stronger hydrodynamic effects for thinner films. Similar is the case for the data available for $18 \mathrm{~nm}$ film (SM Fig. 13). In, ${ }^{10}$ a clear explanation of the observed anomalous value of $\alpha=2$ in experiments could not be provided although, curiously, it was stated that molecular dynamics simulations with full hydrodynamic slip, at the fluid-substrate interface lead to a prediction of $\alpha=2$. The authors, though, commented that in their experimental system full slip was not possible and hence their experimental data was unexplained. We argue that this is very relevant for our system i.e. the observed $\alpha$ value of 2 is clearly indicative of large slip and the reduction of this value with increasing temperature $\mathrm{T}$ corroborates with the anticipated reduction of slip with increasing temperature ${ }^{47,48}$ and also is consistent with the analysis and interpretation we provided to explain the large reduction in viscosity ratios earlier in Fig. 3. Comparison of $\alpha$ for all the films at same temperature (463K) shows an interesting trend $\alpha$ increases with decreasing thickness (SM Fig. 15). This would seem to suggest larger slip at the PGNP-PS interface in thinner films at the same temperature as compared to that in $65 \mathrm{~nm}$ films. This again is consistent with larger viscosity reduction under confinement due to enhanced polymer chain orientational ordering.

In conclusion, we have shown, evidence of strong $\mathrm{HI}$ in dynamics of polymeric nanocolloids in polymer melts and large effective interface viscosity reduction indicative of substantial slip 
at the colloid-polymer interface. We also observe an anomalous hydrodynamic interaction between the PGNPs depending on temperature and film thickness. Hydrodynamic slip at the dewetting PGNP-polymer interface, relevant to our chosen systems, is indicated as the cause of this anomalous power law scaling and large effective viscosity reduction, observed here. Our results throw light, not only on the rich Physics at play in dynamics of these soft colloids in confined polymer melts but also highlights how this could be crucial in determining dispersion and hence all relevant physical properties in such PNC films.

\section{Acknowledgement}

Authors thank the Department of Science and Technology, India for the financial support and Saha Institute of Nuclear Physics, India for facilitating the experiments at the P10 beamline in PETRA-III, DESY, Hamburg, Germany. Authors would also like to thank Venkat Padmanavan, Department of Chemical Engineering, IIT, Kharagpur, India, for useful discussions.

\section{References}

(1) Rubinstein, M.; Colby, R. H. Polymer Physics; Oxford, 2003.

(2) Edwards, S. F.; Doi, M. The theory of polymer dynamics; Clarendon Press Oxford, 1986; Vol. 222.

(3) De Gennes, P. G. Macromolecules 1976, 9, 587-593.

(4) Riese, D. O.; Wegdam, G. H.; Vos, W. L.; Sprik, R.; Fenistein, D.; Bongaerts, J. H. H.; Grübel, G. Phys. Rev. Lett. 2000, 85, 5460.

(5) Bleibel, J.; Domínguez, A.; Günther, F.; Harting, J.; Oettel, M. Soft Matter 2014, 10, $2945-2948$.

(6) Lin, B.; Rice, S. A.; Weitz, D. A. Phys. Rev. E 1995, 51, 423. 
(7) Zahn, K.; Méndez-Alcaraz, J. M.; Maret, G. Phys. Rev. Lett. 1997, 79, 175.

(8) Rinn, B.; Zahn, K.; Maass, P.; Maret, G. Europhys. Lett. 1999, 46, 537.

(9) Diamant, H. J. Phys. Soc. Japan 2009, 78.

(10) Lin, B.; Cui, B.; Xu, X.; Zangi, R.; Diamant, H.; Rice, S. A. Phys. Rev. E 2014, 89, 022303.

(11) Pesche, R.; Nagele, G. Phys. Rev. E 2000, 62, 5432-5443.

(12) Diamant, H.; Cui, B.; Lin, B.; Rice, S. A. J. Phys. Cond. Matt. 2005, 17, S2787-S2793.

(13) Squires, T. M.; Quake, S. R. Rev. Modern Phys. 2005, 77, 977.

(14) Rothstein, J. P.; McKinley, G. H. Phys. Fluids (1994-present) 2001, 13, 382-396.

(15) Reiter, G.; Khanna, R. Langmuir 2000, 16, 6351-6357.

(16) Bäumchen, O.; Jacobs, K. J. Phys.: Cond. Matt. 2010, 22, 033102.

(17) Bäumchen, O.; Fetzer, R.; Jacobs, K. Phys. Rev. Lett. 2009, 103, 247801.

(18) Tuteja, A.; Mackay, M. E.; Narayanan, S.; Asokan, S.; Wong, M. S. Nano Lett. 2007, 7, 1276-1281.

(19) Guo, H.; Bourret, G.; Corbierre, M. K.; Rucareanu, S.; Lennox, R. B.; Laaziri, K.; Piche, L.; Sutton, M.; Harden, J. L.; Leheny, R. L. Phys. Rev. Lett. 2009, 102, 075702.

(20) Ramakrishnan, S.; Shah, S. A.; Ruggeri, L.; Chen, Y. L.; Schweizer, K. S.; Zukoski, C. F. Langmuir 2009, 25, 10507-10514.

(21) Kim, S. Y.; Zukoski, C. F. Soft Matter 2012, 8, 1801-1810.

(22) Kumar, S. K.; Jouault, N.; Benicewicz, B.; Neely, T. Macromolecules 2013, 46, 31993214. 
(23) Srivastava, S.; Basu, J. K. Phys. Rev. Lett. 2007, 98, 165701.

(24) Green, P. F. Soft Matter 2011, 7, 7914-7926.

(25) Bansal, A.; Yang, H.; Li, C.; Cho, K.; Benicewicz, B. C.; Kumar, S. K.; Schadler, L. S. Nat. Mat. 2005, 4, 693-698.

(26) Mackay, M. E.; Tuteja, A.; Duxbury, P. M.; Hawker, C. J.; Van Horn, B.; Guan, Z.; Chen, G.; Krishnan, R. S. Science 2006, 311, 1740-1743.

(27) Balazs, A. C.; Emrick, T.; Russell, T. P. Science 2006, 314, 1107-1110.

(28) Chandran, S.; Begam, N.; Padmanabhan, V.; Basu, J. K. Nat. Comm. 2014, 5, 3697.

(29) Begam, N.; Chandran, S.; Biswas, N.; Basu, J. K. Soft Matter 2015, 11, 1165-1173.

(30) Koga, T.; Jiang, N.; Gin, P.; Endoh, M. K.; Narayanan, S.; Lurio, L. B.; Sinha, S. K. Phys. Rev. Lett. 2011, 107, 225901.

(31) Kandar, A. K.; Srivastava, S.; Basu, J. K.; Mukhopadhyay, M. K.; Seifert, S.; Narayanan, S. J. Chem. Phys. 2009, 130, 121102.

(32) Chandran, S.; Sarika, C. K.; Kandar, A. K.; Basu, J. K.; Narayanan, S.; Sandy, A. J. Chem. Phys. 2011, 135, 134901.

(33) Kim, H.; Rühm, A.; Lurio, L. B.; Basu, J. K.; Lal, J.; Lumma, D.; Mochrie, S. G. J.; Sinha, S. K. Phys. Rev. Lett. 2003, 90, 068302.

(34) Orsi, D.; Fluerasu, A.; Moussad, A.; Zontone, F.; Cristofolini, L.; Madsen, A. Phys. Rev. E 2012, 85, 1-9.

(35) Banchio, A. J.; Nägele, G. J. Chem. Phys. 2008, 128, 104903.

(36) Grabowski, C. A.; Adhikary, B.; Mukhopadhyay, A. Appl. Phys. Lett. 2009, 94, 021903.

(37) Supporting Information. 
(38) Li, C.; Koga, T.; Li, C.; Jiang, J.; Sharma, S.; Narayanan, S.; Lurio, L. B.; Hu, X.; Jiao, X.; Sinha, S. K.; Billet, S.; Sosnowik, D.; Kim, H.; Sokolov, J. C.; Rafailovich, M. H. Macromolecules 2005, 38, 5144-5151.

(39) Wyart, F. B.; De Gennes, P. G. Euro. Phys. J. E 2000, 1, 93-97.

(40) Mackay, M. E.; Dao, T. T.; Tuteja, A.; Ho, D. L.; Van Horn, B.; Kim, H.-C.; Hawker, C. J. Nat. Mat. 2003, 2, 762-766.

(41) Bhattacharyya, S.; Bagchi, B. J. Chem. Phys. 1997, 106, 1757-1763.

(42) Egorov, S. A. J. Chem. Phys. 2011, 134, 084903.

(43) Ganesan, V.; Pryamitsyn, V.; Surve, M.; Narayanan, B. J. Chem. Phys. 2006, 124, $1-4$.

(44) Yamamoto, U.; Schweizer, K. S. J. Chem. Phys. 2013, 139, 064907.

(45) Fetzer, R.; Rauscher, M.; Münch, A.; Wagner, B. A.; Jacobs, K. Europhys. Lett. 2006, $75,638$.

(46) Saugey, A.; Joly, L.; Ybert, C.; Barrat, J.-L.; Bocquet, L. J. Phys.: Cond. Matt. 2005, 17, S4075.

(47) Ichiki, K.; Kobryn, A. E.; Kovalenko, A. J. Comp. Theo. Nanosc. 2008, 5, 2004-2021.

(48) Servantie, J.; Müller, M. Phys. Rev. Lett. 2008, 101, 026101.

(49) Wang, M.; Hill, R. J. Soft Matter 2009, 5, 3940-3953.

(50) Joly, L.; Ybert, C.; Bocquet, L. Phys. Rev. Lett. 2006, 96, 046101.

(51) Lauga, E.; Squires, T. M. Physics of Fluids 2005, 17, 103102.

(52) Pierce, F.; Sorensen, C. M.; Chakrabarti, A. Phys. Rev. E 2006, 74, 021411. 
(53) Chandran, S.; Begam, N.; Basu, J. K. J. Appl. Phys. 2014, 116, 222203.

(54) Kar, G. P.; Begam, N.; Basu, J. K.; Bose, S. Macromolecules 2014, 47, 7525-7532.

(55) Pastorino, C.; Binder, K.; Muller, M. Macromolecules 2008, 42, 401-410. 